\title{
LA ACREDITACIÓN DE LOS PROGRAMAS EDUCATIVOS EN LAS UNIVERSIDADES PÚBLICAS
}

Oscar Priego Hernández*, María Eugenia de la Rosa Leal**, Miguel Ángel Ramírez Martínez***

*Doctor en Finanzas Públicas. Profesor-Investigador de la Universidad Juárez Autónoma de Tabasco.

**Doctora María Eugenia de la Rosa Leal. Profesora-Investigador de la Universidad de Sonora.

***Doctor en Antropología Social. Profesor-Investigador de la Universidad Juárez Autónoma de Tabasco.

Dirección para recibir correspondencia: priegooscarh@hotmail.com

Fecha de recibido: 4 de Marzo de 2017

Fecha de aceptación: 12 de Abril de 2017 


\section{RESUMEN}

Objetivo: Identificar los nuevos roles que deben jugar los docentes y los alumnos en el proceso de la acreditación de los programas educativos de licenciatura en las áreas económico administrativas.

Material y método: El método de estudio que se propone es analítico y descriptivo basado en la experiencia y aplicación de entrevistas personales a profesores, alumnos y personal administrativo. La metodología utilizada es de enfoque cualitativa.

Resultados: Los resultados que se presentan son preliminares en virtud de que la investigación está en proceso y muestran que los profesores y alumnos en las áreas económico administrativas no están debidamente informados de la verdadera filosofía del significado de un programa de estudio acreditado.

Conclusiones: Las universidades que han logrado la acreditación de sus programas educativos deben diseñar estrategias para difundir los resultados entre sus profesores y alumnos así como evaluar las ventajas de los nuevos roles y compromiso en el proceso de acreditación.

Palabras clave: Calidad. Evaluación. Acreditación. Educación Superior. 


\section{INTRODUCCIÓN}

En un mundo globalizado en el que se desenvuelven las Instituciones de Educación Superior (IES), es necesario ser competitivos. En México, se mantiene una política de modernización educativa orientada a elevar la eficiencia y eficacia de los Programas Educativos dando origen a la evaluación de la calidad en la educación superior en función a los resultados, debido a que actualmente, los recursos ya no son asignados de manera equitativa, sino que existe una bolsa concursable en función al desempeño institucional.

Las IES como ejes motores del desarrollo económico, social y cultural del país deben estar inmersas en la dinámica de la evaluación para ser competitivas; sin embargo, a pesar de ser la acreditación uno de los objetivos estratégicos del Plan Nacional de Desarrollo (PND 2013-2018), todavía es muy reducido el número de IES que cuentan con este reconocimiento. Es evidente identificar los cambios que se generan derivados de la acreditación de sus programas y difundirlos con el propósito de motivar a otras instituciones a implementar esta práctica.

Este trabajo tiene como objetivo identificar las fortalezas de las áreas económico administrativas con la finalidad de mejorar la calidad educativa de nuestro país para que a su vez, incida en una mejora en el desempeño profesional de los docentes y la formación integral de los egresados que permita incrementar la competitividad en nuestro entorno. La importancia de esta investigación, radica en su contribución a la generación del conocimiento científico dentro del campo de la investigación educativa, así como a la construcción de aprendizajes.

La evaluación de los programas académicos es una de las herramientas más importantes para mejorar la calidad de la educación, por ello es necesario establecer estrategias y acciones que permitan difundir en las IES las mejores prácticas existentes a nivel nacional e internacional para hacer de la evaluación una cultura. Uno de los retos que se presentan en el corto plazo, está el de iniciar los trabajos de acreditación de los nuevos programas educativos basados en competencias; en donde el involucramiento y compromiso de los docentes y alumnos será determinante para lograrlo.

En este sentido, si se tuviera que elegir un lema que guiara las metas y propósitos de la escuela del siglo XXI, sin duda el más aceptado a estas alturas entre educadores e investigadores, políticos que toman decisiones sobre la educación e intelectuales que reflexionan sobre ella, sería el que la educación tiene que estar dirigida a ayudar a los alumnos a aprender a aprender. De

PRIEGO-HERNÁDEZ O, DE LA ROSA-LEAL, M.E., RAMÍREZ-MARTÍNEZ M.A. 
hecho, es difícil encontrar alguna reflexión sobre el futuro de la educación, ya sea básica (Kirk, 1986) o universitaria (Michavilla \& Calvo, 1998) hecha desde un planteamiento pedagógico (Bruner, 1997); filosófico (Savater, 1997); o bien, de políticas educativas de la (UNESCO-OCDEANUIES) que no afirme enfáticamente que una de las funciones de la educación futura debe ser: "Promover la capacidad de los alumnos de gestionar sus propios aprendizajes, adoptar una autonomía creciente en su carrera académica y disponer de herramientas intelectuales y sociales que les permitan un aprendizaje continuo a lo largo de toda su vida" (Pozo y Monereo, 1999).

Ante esta problemática, las universidades públicas han tenido que reorientar sus políticas y diseñar estrategias dirigidas a obtener financiamiento de recursos mediante fuentes alternas. Para ello, ahora es necesario recurrir a la búsqueda de alternativas distintas a las actuales (subsidios federales y estatales) por otro tipo de financiamiento basado en esquemas de indicadores de calidad (UNESCO, 1992) tales como la autoevaluación por organismos externos y el fortalecimiento de los programas educativos dirigidos a generar recursos propios mediante programas autofinanciables.

Al mismo tiempo, han tenido que realizar los ajustes necesarios en materia de gasto y asumir de manera conjunta el compromiso con la comunidad universitaria compuesta por profesores, investigadores, administrativos, sindicatos y alumnos. En este proceso de ajustes los profesores y alumnos deben asumir el nuevo rol que les corresponde, verdaderamente importante en este compromiso marcado por la escasez de recursos para la educación. Uno de estos mecanismos, es continuar con la evaluación que realizan los organismos externos para la acreditación de los programas educativos, la cual es solicitada de forma voluntaria por las IES.

\section{OBJETIVO}

Identificar los nuevos roles que deben jugar los docentes y alumnos en el proceso de la Acreditación de los Programas Educativos de licenciatura en las áreas económico administrativas de una Universidad Pública para establecer estrategias de mejora en el proceso de enseñanzaaprendizaje.

PRIEGO-HERNÁDEZ O, DE LA ROSA-LEAL, M.E., RAMÍREZ-MARTíNEZ M.A. 


\section{ESTUDIO DE LA PROBLEMÁTICA}

\section{Antecedentes}

México es actualmente uno de los países miembros de la Organización para la Cooperación del Desarrollo Económico (OCDE) que ha enfrentado una de las peores crisis económicas de los últimos 80 años. Situación ocasionada por diversos factores externos entre otros principalmente los derivados por la crisis financiera ocurrida recientemente en los Estados Unidos y por otro, debido la caída de los precios internacionales del petróleo crudo en más del $50 \%$ durante el año 2015 que ha afectado considerablemente las finanzas públicas de nuestro país. Este fenómeno sin duda, ha sido un factor determinante que afecta el sano desarrollo de los Programas Educativos y el cumplimiento de las metas e indicadores que se tenían previstos a mediano plazo.

Esta situación por demás alarmante ha obligado a las autoridades financieras responsables de la asignación y distribución de los recursos públicos a efectuar los recortes pertinentes al gasto público en educación. Lo anterior, también se refleja en los recortes presupuestales anunciados recientemente por las autoridades educativas, lo cual indica que estamos ante una escasez de recursos públicos generalizada por la contracción de la economía mexicana lo cual impacta directamente en el desarrollo de los programas educativos en las universidades públicas.

Por su parte, debe entenderse que, en este nuevo siglo, el desarrollo social y el crecimiento económico están directamente relacionados con el perfeccionamiento y la pertinencia de su sistema de educación superior, lo que origina que las universidades sean instituciones autónomas y criticas conocedoras de las necesidades y movimientos del entorno, de las demandas laborales y de las interacciones y desarrollo en contextos internacionales (Roa Valero, 2008)

Para introducirse en la temática de la educación superior en México, es conveniente hacerse la siguiente pregunta de investigación: ¿La acreditación de los Programas Educativos en las universidades públicas ha sido realmente un instrumento para mejorar los indicadores de calidad de la educación superior, fortalecer la formación integral de los alumnos y las labores académicas de los profesores? La problemática que se plantea en este trabajo está relacionada con el proceso para la Acreditación de los Programas Educativos de licenciatura que realizan los organismos acreditadores: el Consejo de Acreditación de la Enseñanza de la Contaduría y Administración 
(CACECA) y el Consejo Nacional de Acreditación de la Ciencia Económica (CONACE) en las Universidades Públicas.

Al respecto, en materia de educación media y superior consideramos que México está viviendo una paradoja ya que siendo uno de los países miembros de la OCDE que invierte cantidades considerables de recursos en este rubro, resulta que hoy todavía muestra bajos indicadores relacionados con la calidad de la educación en el contexto internacional. Por su parte, cuando el análisis se refiere al panorama en Latinoamérica, la situación cambia debido a la similitud en las economías y problemas comunes que comparten estos países.

La educación es la base fundamental para que un país pueda alcanzar su desarrollo económico; por ello, la inversión en este renglón es una prioridad para mejorar los indicadores que tienen que ver con la calidad de vida de los ciudadanos de los países desarrollados que invierten en educación. México es uno de los países que en los últimos años ha realizado grandes esfuerzos por alcanzar mejores indicadores de calidad en materia de educación superior.

Los niveles de educación superior de un país, están estrechamente relacionados con los hábitos de vida de la población que se refleja en aspectos que tienen que ver con rezago social, económico y de bienestar social. En este sentido, la educación superior en México está fuertemente vinculada con los indicadores de desarrollo.

Es evidente, como lo señala Espinoza Marum (2003) que existe un gran reto en cuanto a la cobertura de la educación superior para asegurar los principios de equidad y pertinencia, pues su tasa del $20 \%$ se ubica entre una de las más bajas del continente, se ha considerado ineludible, además, el reto de la calidad. Necesita mejorar sustancialmente varios aspectos básicos del sistema educativo que permitan incrementar los indicadores de calidad que exige el nuevo entorno educativo en el contexto internacional del siglo XXI. También es cierto que, si retomamos las estadísticas de la matrícula de estudios superiores y posgrado de los años ochenta, se observa que ha tenido un incremento significativo, sin embargo, hoy los indicadores muestran la situación de la educación superior con serias deficiencias.

Este trabajo, tiene la finalidad de realizar un análisis de la situación que presenta el proceso de Acreditación de los Programas Educativos en las universidades de educación superior del Estado de Tabasco. El método de estudio utilizado es analítico y descriptivo mediante un modelo 
deductivo que implica ordenar las ideas que van de lo general a lo particular en el análisis de la temática abordada.

Ante el panorama planteado, las autoridades educativas en México están adoptando estrategias y medidas administrativas basadas en la cultura de la evaluación que permitan mejorar la calidad de los sistemas educativos. Hay que recordar, que la calidad de una institución educativa se mide por la calidad de sus egresados, que a su vez tiene que ver con el trabajo que realizan los docentes y los otros actores para ofrecer a la sociedad profesionistas cada vez mejor preparados.

De conformidad con la propuesta de la ANUIES, se establece que "en el horizonte de las dos primeras décadas del siglo XXI, la misión del Sistema de Educación Superior es lograr el mejoramiento del conjunto y de cada una de sus instituciones, gracias al compromiso de sus miembros en procesos rigurosos y permanentes de evaluación y aseguramiento de la calidad", (Rodríguez, R.: 2000).

Al respecto, ante este gran reto para el año 2020, hoy la mayoría de las instituciones públicas de educación superior están adaptando y reorientando sus planes de estudios "tradicionales" hacia modelos educativos de calidad que garanticen la formación integral, permanencia y egreso de los estudiantes. Esto implica sin duda, un gran esfuerzo por avanzar hacia la calidad de la educación de las instituciones públicas; proceso que requiere de un serio compromiso de responsabilidad de los actores involucrados: institución, profesores, alumnos y personal administrativo. En la medida en que estos actores asuman su compromiso con la calidad, los resultados de la acreditación podrán evaluarse de manera cualitativa y cuantitativamente.

Las universidades públicas en este nuevo siglo enfrentan serios y graves problemas, sin duda que la falta de recursos afecta de manera directa las funciones adjetivas y sustantivas e impacta en el desarrollo de los programas educativos y en la formación integral de los estudiantes. Bajo este panorama de retos y desafíos de la educación superior en México es pertinente reflexionar si realmente la acreditación de los programas educativos mejora los indicadores de calidad de la educación superior, permite fuentes de financiamiento alternas, fortalece la formación integral de los alumnos y las labores académicas de los profesores.

Por ello, se considera necesario que los responsables de la administración de las IES, diseñen estrategias que permitan evaluar de manera continua el proceso de la acreditación para detectar

PRIEGO-HERNÁDEZ O, DE LA ROSA-LEAL, M.E., RAMÍREZ-MARTÍNEZ M.A. 
las principales oportunidades y amenazas para establecer estrategias que apoyen a los profesores a mejorar las tareas docentes en busca de la calidad educativa.

\section{Marco teórico}

En esta investigación, es focalizado el paradigma de la Administración por calidad de Edward Deming, quien establece que través de cuatro fases orientadas a la búsqueda constante de la perfección, se llega a la excelencia, denominadas ciclo de Mejora continua, ciclo de Deming o ciclo PHVA (Planear, Hacer, Verificar y Actuar), representando la verificación o evaluación una fase indispensable en todo proyecto, no algo complementario que solo haremos si nos sobra tiempo.

Las contribuciones del Dr. Deming y el ciclo de diseño de Shewart, interpretado posteriormente por Mizuno como el círculo de control PHVA en los años cincuenta han tenido una gran influencia partir de los noventas en las instituciones educativas. (Apodaca y Lobato, 1997). La Teoría Deming se ha extendido en la actualidad hasta convertirse en una filosofía de administración, que es impulsada por la mejora continua misma que responde a las necesidades y expectativas del cliente dónde su objetivo es crear una organización comprometida con la calidad. Su filosofía es un importante marco para implementar el mejoramiento de la calidad y la productividad. (Herrera, 2007).

\section{MATERIAL Y MÉTODO}

El método de estudio que se propone es analítico y descriptivo basado en la aplicación de entrevistas personales a profesores, alumnos y personal administrativo en una Universidad Pública. En la investigación también será utilizado el método analítico para la revisión de la literatura y el análisis de la información estadística del tema sobre las fortalezas y debilidades que representa la Acreditación de los Programas Educativos de licenciatura en las IES. Se identificó la problemática mediante un modelo deductivo, procurando presentar las ideas con el criterio que va de lo general a lo particular de conformidad con la temática abordada. El trabajo consiste, en analizar los instrumentos de evaluación utilizados durante el proceso de la Acreditación que realiza el CACECA y el CONACE.

La investigación que se realiza es de tipo cualitativo y exploratoria (Hernández, Fernandez y Batipsta 2010) mediante la aplicación de la técnica de diagnóstico con encuestas a través de 
cuestionarios de preguntas abiertas y cerradas, que permitan la recolección de información y el análisis documental de la bibliografía relacionada con este tema. Consiste en utilizar el análisis como instrumento principal para recabar la información mediante la aplicación de la técnica de diagnóstico y el análisis de la bibliografía especializada relacionada con este tema. El estudio está basado en la aplicación de entrevistas, el análisis de indicadores, el uso de estadísticas, así como el uso de Internet. Estos instrumentos incluyen además técnicas de investigación como entrevistas realizadas a los involucrados en la actividad docente.

\section{Evaluación y Acreditación}

Históricamente, antes de que llegara la revolución promovida por Ralph W. Tyler, en Francia, se inició en los años veinte un primer acercamiento a la verdadera evaluación educativa. En 1942 Smith y Tyler plantean la necesidad de una evaluación científica que sirva para perfeccionar la calidad de la educación. (Escudero, 2003). Medio siglo después de que Tyler revolucionara el mundo de la evaluación educativa, se observa la fortaleza, coherencia y vigencia de su pensamiento, pues a pesar de que las reformas Tyleriana no siempre se aplicaron de inmediato, en España, los planteamientos de Tyler se extendieron con la Ley General de Educación de 1970.

La evaluación pretende alcanzar un nivel de comprensión sobre el funcionamiento del programa en su contexto, fundamentalmente si es una reflexión sistemática, tiene que ser la base de las innovaciones que se introduzcan (Casanova, 1992).

Las acciones que en materia de evaluación de la educación superior se han emprendido en México datan, principalmente, de finales de los años setenta del siglo XX, y emanan de los programas de gobierno nacionales y de iniciativas de la ANUIES. La evaluación se institucionalizó con el Programa para la Modernización Educativa 1989-1994, del gobierno federal, en que el concepto de modernización de la educación se concibe en términos de calidad, eficiencia, cobertura e innovación de la misma.

El Programa de Desarrollo Educativo 2001-2006, con el propósito de fundamentar el diseño de políticas y la toma de decisiones dirigidas al mejoramiento de la calidad y la equidad de la educación básica, puso en marcha en el 2002 el Acuerdo Social por la Calidad de la Educación, cuyo primer punto estratégico fue la Creación del Instituto Nacional de Evaluación Educativa. En el PND 2007-2012, uno de los ejes rectores, era elevar la calidad educativa, por lo que se debe 
hacer cambios en la práctica evaluativa que permitan hacer de la Educación el Gran Proyecto Nacional que allí se contempla (Franyuti, 2010).

Entre los indicadores y las metas del Programa Sectorial de Educación, se consideraba para el año 2012: Incrementar a 60\% el porcentaje de alumnos en programas reconocidos por su buena calidad (nivel 1 CIEES o acreditados COPAES). Para el año 2016 estos indicadores se han incrementado considerablemente en todas las universidades públicas. El objetivo para el año 2020 es contar con el 100\% de los Programas Educativos con la calidad de estos indicadores.

La acreditación, en la mayoría de los países es un proceso voluntario, ejecutado por pares por medio de agencias, generalmente no gubernamentales, y tiene como propósito garantizar públicamente la calidad de los servicios académicos que ofrece una institución, certificar condiciones apropiadas para recibir subsidios públicos, aportes privados, facilitar la movilidad estudiantil, promover el mejoramiento de instituciones y programas. La acreditación de programas académicos tiene su antecedente inmediato en los procesos de evaluación que adquirieron importancia creciente en el mundo a partir de la década de los años ochenta. Ello se ha manifestado en las políticas y programas que han emprendido los gobiernos de todo el orbe, apoyados por organismos internacionales como la Organización de las Naciones Unidas para la Educación, la Ciencia y la Cultura (UNESCO) y la Organización para la Cooperación y Desarrollo Económico (OCDE), entre otras.

La elección de un modelo evaluativo, supone elegir una teoría epistemológica y de concepción de la realidad. La teoría de evaluación educativa base en esta investigación, está sustentada en el modelo de Evaluación etnográfica de Santos Guerra; quien enfatiza las bondades de la teoría de evaluación del currículo propuesta por Tyler para sustentar la práctica de la evaluación como factor clave de cambio en las universidades. Implica conocer de qué manera funcionan las escuelas para comprender y mejorar las actuaciones. Este enfoque tiene en cuenta aspectos como el contexto diacrónico y sincrónico, los intercambios sociales, una investigación interpretativa de los hechos, la consideración de los procesos, combinar la evaluación interna y externa.

\section{Modelo de Evaluación para la Acreditación del (CACECA)}

El modelo de evaluación considerado en esta investigación, consiste en una evaluación integral, primeramente, la institución se autoevalúa con el instrumento proporcionado por el organismo 
acreditador y posteriormente con el apoyo de pares académicos se da la evaluación en sitio, documental y mediante recorrido por la institución, incluyendo entrevistas con profesores y alumnos. Establece que la acreditación tiene una vigencia de 5 años, durante los cuales se dan visitas de seguimiento para verificar el cumplimiento y avance de las recomendaciones. Los criterios que evalúa se encuentran distribuidos en 8 variables: a) Profesores, b) Estudiantes, c) Modelo Académico, d) Formación Integral, e) Recursos Financieros, f) Recursos-Eficiencia, g) Vinculación h) Información Adicional.

EI CACECA es uno de los 26 organismos no gubernamentales, especializados y facultados para acreditar la calidad de los programas académicos que se imparten en las instituciones de educación superior en sus diferentes disciplinas en México siendo el único facultado para acreditar programas académicos de contaduría, administración y áreas afines.

Para poder hacer frente a los retos que presenta hoy en día la globalización es necesario ser competitivos, y eso se logra haciendo las cosas bien a la primera; es decir, implementando la calidad como habito de vida, y la calidad comienza con educación y termina con educación, sin la existencia de las IES sería difícil el avance y progreso de la humanidad, por eso la necesidad de evaluación de la calidad de los programas académicos que permita conocer las fortalezas y debilidades para la implementación de planes estratégicos de acción que les permitan día con día ser reconocidas como las mejores por todos y cada uno de sus clientes.

La propuesta de hacer de la evaluación una cultura, es una oportunidad, para que las IES acepten el reto y a partir de la identificación de sus fortalezas y debilidades por parte de pares evaluadores externos, diseñen estrategias que le permitan hacer cambios planificados que las lleven a ser mejor cada día y entregar a la sociedad egresados que les permitan enfrentar los retos de un mundo globalizado.

Es necesario identificar el rol que juegan los docentes y alumnos en las actividades que integran el proceso de evaluación para la acreditación de programas educativos y en la integración de las evidencias de los criterios o indicadores que deben cumplir las IES para obtener este reconocimiento por la calidad educativa.

La calidad es considerada como detonador para el desarrollo de un país, formando parte de los objetivos del Plan Nacional de Desarrollo 2013-2018; por ello, la educación en este sentido ha 
tenido un crecimiento, formando parte de los planes y programas de estudios de las instituciones públicas de educación de nivel superior, requiriendo además que estos a su vez sean de calidad. Sin embargo, todavía existe escepticismo con respecto a la calidad, para algunos, la calidad sigue siendo una utopía o falacia; sin embargo, W. Edward Deming en los años cincuenta inicio un movimiento conocido como filosofía de Mejora continua, orientada a la búsqueda constante de la perfección, en donde a través de cuatro fases se llega a la excelencia.

Hoy en día, todas las IES como los demás organismos sociales, buscan ser excelentes, competitivas, de clase mundial y para ello es necesario contar con recursos, estrechamente interrelacionados y bien liderados. Estos recursos (humanos, tecnológicos, materiales y financieros deben de ser eficaces, eficientes y competitivos, sin embargo, son escasos y están bien cotizados, por lo que las instituciones hacen todo lo necesario para poder tener acceso a ellos, mediante diversos proyectos concursables 100\% como lo fue en su oportunidad el Programa Integral para el Fortalecimiento Institucional.

En el mundo globalizado en donde las competencias trascienden fronteras y el conocimiento humano avanza a pasos agigantados, la evaluación y la acreditación son instrumentos de gran valía, que nos permiten determinar la relevancia, pertinencia y sobre todo competitividad social la de nuestros programas de estudio y en consecuencia de nuestras Instituciones y quienes colaboran en ella; sin embargo aún no se ve la evaluación como una estrategia para la mejora continua, no se puede mejorar lo que no se mide; es decir, se necesitan indicadores no solo cuantitativos sino cualitativos, que muestren cómo estamos con respecto a lo planificado, para tomar decisiones, e implementar acciones que nos lleven al cumplimiento de los objetivos institucionales orientados a la satisfacción de los clientes internos y externos.

\section{RESULTADOS}

Los resultados preliminares de este estudio muestran que los profesores y alumnos de las áreas económico administrativas no comprenden la verdadera filosofía que significa un programa educativo acreditado. Algunos hallazgos del estudio se presentan a continuación:

$>$ La falta de compromiso para lograr la calidad de los Programas Educativos que hoy requieren las universidades públicas.

$>$ La falta de difusión adecuada de los resultados de la acreditación por parte de la IES.

PRIEGO-HERNÁDEZ O, DE LA ROSA-LEAL, M.E., RAMÍREZ-MARTÍNEZ M.A. 
La Acreditación de los Programas Educativos, es una estrategia que seguirá siendo un instrumento valioso de evaluación para que las universidades transiten hacia los indicadores de calidad.

$>$ Las IES pueden acceder a fondos federales que significan recursos adicionales para mejorar sus procesos de calidad mediante indicadores.

\section{CONCLUSIONES}

Las IES necesitan fortalecer una cultura de evaluación para la mejora de la calidad educativa que permita el estudio de los efectos, pudiendo ser, los beneficios, problemas y recomendaciones derivadas del proceso de acreditación.

La acreditación es una autoevaluación que permite posicionar a las universidades públicas mediante el logro de indicadores de calidad en programas acreditados que muestra las fortalezas y debilidades para el seguimiento de las recomendaciones que realizan los organismos acreditadores.

Es recomendable evaluar de manera permanente las tareas docentes del profesor para lograr mayor impacto en la formación integral de los alumnos de los programas educativos acreditados y diseñar estrategias que permitan evaluar las ventajas de los nuevos roles de los docentes y alumnos en este proceso en donde todos los actores deben estar involucrados en particular los profesores y alumnos de los programas educativos acreditados. 


\section{REFERENCIAS BIBLIOGRÁFICAS}

ANUIES (1995). Declaración de pertinencia. México: ANUIES.

Apodaca, P. y Lobato, C. (1997). Calidad en la universidad: orientación y evaluación. Barcelona: Alertes.

Bruner, J. (1997). La educación puerta de la cultura. Madrid: Visor.

CACECA. (2006). Consejo de Acreditación de la Enseñanza de la Contaduría y Administración. México: CACECA.

CACECA. (Enero de 2007). Modelo de Evaluacion para la Acreditación. Ciudad de México, D.F., México. CACECA. (Recuperado el 30 de Junio de 2010, de www.caceca.org)

Casanova, M. A. (1992). La evaluación, garantía de calidad para el centro educativo. Zaragoza: Edelvives.

COPAES. (2006,3). Obtenido de Marco General para los Procesos de Acreditación de Programas Académicos de Nivel Superior: http://www.copaes.org.mx/home/docs/docs_acred/3_Marco_general.pdf

COPAES. (2006,9). Obtenido de: Escudero, Tomás (2003). Desde los tests hasta la investigación evaluativa actual. Un siglo, el $X X$, de intenso desarrollo. Revista Electrónica de Investigación y Evaluación Educativa, 9(1). http://www.uv.es/RELIEVE/v9n .

Espinosa E.M. (2003). Innovación en la docencia de pregrado. Un reto de la calidad y la acreditación en México. Universidad. Unión de Universidades de América Latina, España, Portugal, 26, p. 23.

Franyuti, M. d. (9 de Agosto de 2010). "La Evaluación Educativa en México" . Villahermosa, Tabasco, Mexico.

Hernández, Fernández, y Baptista (2010). Metodología de la Investigación (5ed.). México: Mc Graw-Hill. 


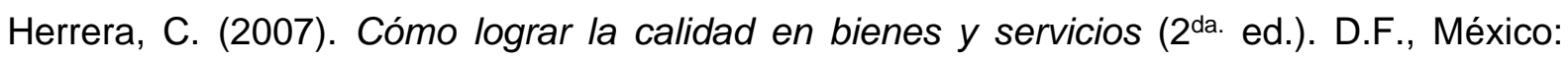
Limusa.

Kirk, K. (23 de noviembre de 1986). Searle scholars program. Recuperado el 12 de marzo de 2006, de SCHOLAR PROFILE: http://www.searlescholars.net/person/360

Michavilla, F., \& Calvo, B. (1998). La Universidad Española hoy. Propuestas de una política universitaria. España: Sintesis.

Organización para la Cooperación de Desarrollo Económico (OCDE). (19 de septiembre de 2007). Recomienda OCDE privatizar la educación superior. Artículo publicado en el periódico la Jornada. México.

Pozo J. y Monereo C. (Coordinadores) (1999). El aprendizaje estratégico. Madrid, España: Aula XXI, Santillana.

Roa Valero, A. (2008). Hacia un modelo de aseguramiento de la calidad en la educación superior en Colombia: estándares básicos y acreditación de excelencia. Cartagena: Consejo Nacional de Acreditación de Colombia.

Rodríguez, R. (2000). La educación superior en el siglo XXI. Líneas estratégicas de desarrollo. Una propuesta de la ANUIES. Revista de la Educación Superior ANUIES, 14(113).

Savater, F. (1997). El valor de educar. Barcelona: Ariel.

UNESCO. (1992). Organización de las Naciones Unidas para la Educación, la Ciencia y la Cultura, CRESAL. París: UNESCO. 